\title{
3,4-桥环吲哚类生物碱的合成研究进展
}

\author{
单 冬贾彦兴* \\ (北京大学药学院 天然药物及仿生药物国家重点实验室 北京 100191)
}

\begin{abstract}
摘要 取代的吲梠类天然产物是自然界中普遍存在的一类杂环化合物，由于其具有广泛的生理活性，以及相当一部分 作为重要的临床使用药物, 100 多年以来, 吲哚的合成及官能团化一直是有机合成化学家关注的一个重要领域. 在众多 吲哚类生物碱中, 含有 3,4 -桥环呫哚骨架的天然产物占据了相当一部分, 由于其独特的结构和良好的生物活性, 这些分 子引起了有机合成化学家的广泛兴趣. 重点概述了构建 3,4-桥环骨架的主要合成方法和策略, 并对一些方法在天然产 物全合成中的应用作简要介绍.
\end{abstract}

关键词＼cjkstart吲哚生物碱; 3,4-桥环吲哚; 天然产物; 全合成

\section{Progress in Synthesis of 3,4-Fused Indole Alkaloids}

\author{
Shan, Dong Jia, Yanxing* \\ (State Key Laboratory of Natural and Biomimetic Drugs, School of Pharmaceutical Sciences, Peking University, \\ Beijing 100191)
}

\begin{abstract}
The indole nucleus is one of the most important heterocycles for its presence in a vast number of bioactive natural products, pharmaceuticals, and agrochemicals. Due to their widest spectrum of biological activity and some of them have been used in clinical trials, construction and functionalization of indoles have attracted considerable interests from synthetic and medicinal chemists for over a hundred years. Among the naturally occurring indole alkaloids, those containing 3,4-fused indole skeleton possess a major part and have been especially considered attractive synthetic targets because of their unique structure and biological activities. This review emphasized on the methodologies and strategies of construction of 3,4-fused indole skeleton, and their application in total synthesis of natural products.
\end{abstract}

Keywords indole alkaloids; 3,4-fused indoles; natural products; total synthesis

取代的吲哚类天然产物是自然界中普遍存在的一 类杂环化合物, 由于其具有广泛的生理活性, 以及相当 一部分作为重要的临床使用药物, 100 多年以来, 吲哚 的合成及官能团化一直是有机合成化学家关注的一个 重要领域 ${ }^{[1]}$.

在众多吲哚类生物碱中, 有一类比较有特点的 3,4桥环吲哚衍生物, 如 dehydrobufotenine $(\mathbf{1})^{[2]}$, 麦角酸 (lysergic acid, 2) $)^{[3]}$, welwistatin $(3)^{[4]}$, communesin F (4) ${ }^{[5]}$, dragmacidin E (5) ${ }^{[6]}$, decursivine $(\mathbf{6})^{[7]}$, indolactam V (ILV,

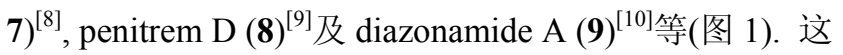
些天然产物在 3,4-位成六元、七元、八元、九元以及更 大的环, 而且在环的不同位置上有碳、氧、氮等不同的
原子取代. 这类含有 3,4-桥环吲哚骨架的天然产物占据 了吲哚类生物碱的相当一部分, 并且具有显著的生理活 性以及作为临床使用药物, 如: 麦角新碱(ergometrine, 10)临床上用于治疗产后子宫出血、子宫复旧不良、月 经过多等; 麦角胺(ergotamine, 11)和咖啡因合用用于治 疗偏头痛; dragmacidin $\mathrm{E}$ 可以作为丝氨酸-苏氨酸蛋白 磷酸酶抑制剂; 而 indolactam V 不仅能够选择性活化蛋 白激酶 C (PKC) 的某些亚族，同时也是一种鼠皮肤癌有 效的启动因子. 除天然产物外，还有一些含有类似骨架 结构并且具有很好生物活性的化合物，如化合物 12 可 以作为降钙素基因相关肽(GCRP)受体拮抗剂，对于治 疗包括偏头痛在内的头痛十分有效.

\footnotetext{
* E-mail: yxjia@bjmu.edu.cn

Received April 22, 2013; revised May 13, 2013; published online May 15, 2013.
}

Project supported by the National Natural Science Foundation of China (Nos. 21290180, 20972007), the National Basic Research Program of China (973 Program) (No. 2010CB833200) and the Ph.D. Programs Foundation of Ministry of Education of China (No. 20120001110100).

国家自然科学基金(Nos. 21290180，20972007)、国家重点基础研究发展规划(973 计划，No. 2010CB833200)和国家教育部博士点基金(No. 20120001110100)资助项目. 


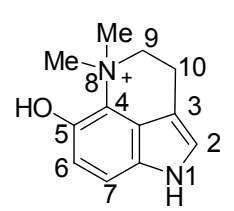

dehydrobufotenine (1)<smiles>C[C@@H]1N=C(N)N[C@]12c1nc(-c3c[nH]c4cc(Br)ccc34)c(=O)[nH]c1-c1c[nH]c3c(O)ccc(c13)[C@@H]2C</smiles>

$\mathrm{Me}$

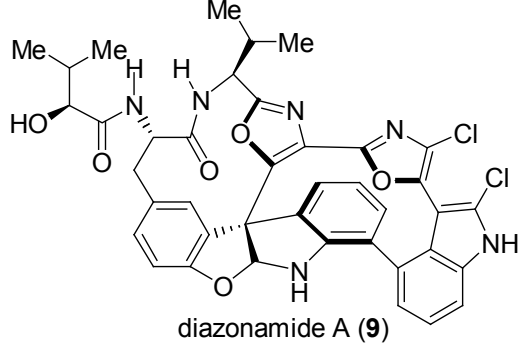<smiles>CN1CC(C(=O)O)C=C2c3cccc4[nH]cc(c34)C[C@H]21</smiles>

lysergic acid (2)<smiles>O=C1NCCc2c[nH]c3ccc(cc23)O[C@H]1c1ccc2c(c1)OCO2</smiles>

decursivine (6)

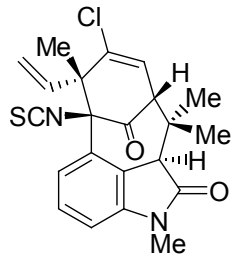

welwistatin (3)

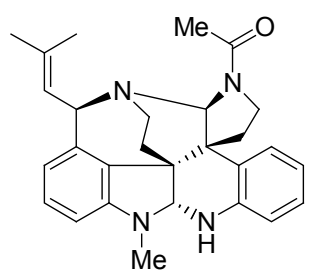

communesin $\mathrm{F}(\mathbf{4})$

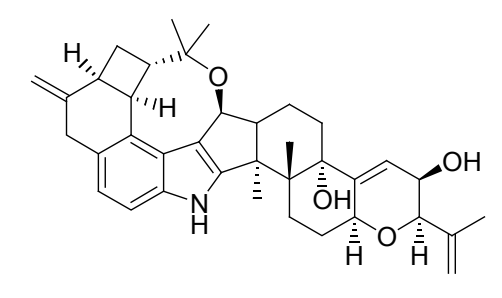

penitrem $\mathrm{D}(\mathbf{8})$

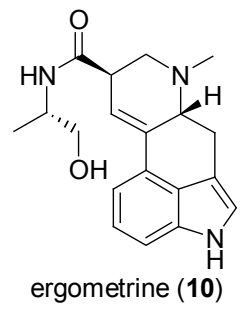<smiles></smiles>

indolactam $\vee(7)$

图 1 几种 3,4 桥环吲哚生物碱

Figure 1 Examples of 3,4-fused indole alkaloids

由于这类含有 3,4-桥环吲哚骨架的天然产物的独特 结构和良好的生物活性, 这些分子早已引起了有机合成 化学家的广泛兴趣, 有机合成化学家们也已经发展了多 种策略来实现它们的合成. 传统的策略绝大多数是从已 有吲哚母核出发, 主要针对于 3,4-桥环的构建. 近几年 来, 一些合成 3,4-桥环吲哚的新方法发展起来. 它们从 不同的位点切断, 通过串联反应, 或一步构建多个环, 实现了更加高效快速的合成. 下面就针对一些有代表性 的 3,4-桥环吲哚的合成策略及其在天然产物全合成中的 应用作一简要的综述.

\section{1 传统的合成 3,4-桥环吲哚骨架的策略}

传统的合成 3,4-桥环吲哚骨架的策略基本都是从已 有吲哚母核出发, 而重点集中在 3,4-桥环的构建上. 通 常的方法可以分为三大类: 第一类是在吲哚母核的 3-位 引入适当的取代基, 最后在 4-位关环; 第二类是在吲哚 母核的 4-位引入适当的取代基后从 3-位关环; 第三类则 是在吲哚母核的 3-位和 4-位分别引入适当的取代基, 最 后在中间关环，从而完成整个分子骨架的构建. 以下是 一些常用的方法.

\section{1 吲哚 4-位没有取代基在吲哚 4-位关环}

\subsection{1 分子内的 Friedel-Crafts 反应}

由于吲哚的 4-位并不是反应活性最高的位点, 因此
应用常规的方法如亲电取代反应很难直接在吲哚的 4位引入取代基. 但通过改变吲哚母核的电性, 位阻, 以 及通过一些络合定位作用等，则可以实现在吲哚不活泼 的 4-位选择性的官能团化.

还原吲哚的 2,3-位双键为吲哚啉, 可以消除活泼的 2-位在反应中的竞争. 而尽管吲哚啉的苯环部分 5-位的 活性高于 4-位, 但作为分子内反应, 3-位丙酸取代的吲 哚啉衍生物 13 可以高选择性地在 4-位进行 FriedelCrafts 酰基化反应, 生成 Konfeld 酮 14, 再将吲哚啉氧化 为吲哚, 即得到 3,4-桥环吲哚的骨架(图 2). Woodward 小 组 ${ }^{[11]}$ 正是应用这一策略完成了麦角酸(2)的首次全合成.

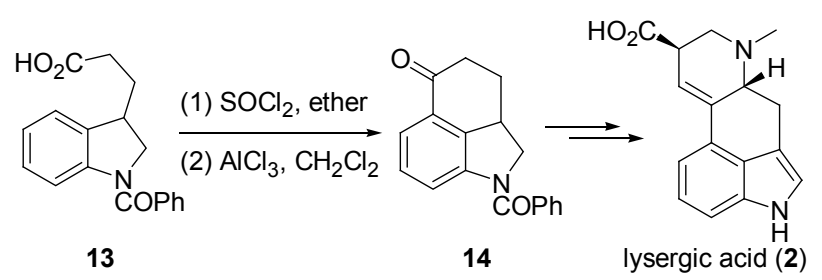

图 2 吲哚啉的 4-位 Friedel-Crafts 反应

Figure 2 Friedel-Crafts reaction on the 4-position of indolines

将吲哚的 2-位进行取代, 从而封闭 2-位反应位点, 也可以使分子内亲电反应选择性地发生在吲哚的 4-位. 如 2-位甲基取代的吲哚衍生物 15 可以在 4-位很顺利地 发生 Friedel-Crafts 酰基化反应，生成 Uhle 酮衍生物 
(16) ${ }^{[12]}$. 而 2-位苯基取代的吲哚衍生物 17 也可以在酸性 条件下经过 $\mathrm{N}$-酰基亚铵离子中间体 19 选择性地在吲哚 的 4-位发生 Friedel-Crafts 反应, 生成 3,4-并环的吲哚氮 杂卓衍生物(20)(图 3) $)^{[13]}$.<smiles>Cc1c(C2CC(=O)OC2=O)c2ccccc2n1C(C)(F)F</smiles>

$$
\underset{\left(\mathrm{CH}_{2} \mathrm{Cl}\right)_{2}}{\stackrel{\mathrm{AlCl}_{3}}{\longrightarrow}}
$$<smiles>[R]C(=O)C([R])CC(=O)NCCc1c(-c2ccccc2)[nH]c2ccccc12</smiles>

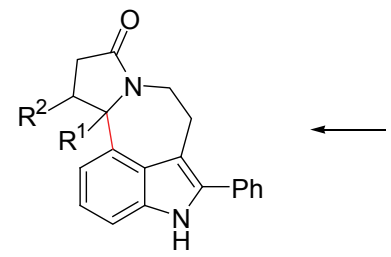

20

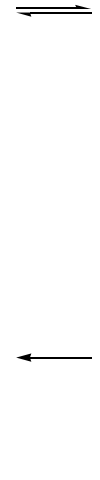

32 -取代吲哚的分子内亲电反应

Figure 3 Intramolecular electrophilic reaction of 2-substituted indoles

另外, 在 Friedel-Crafts 反应中, 还可以通过大位阻 的保护基和试剂的选择来提高 4-位选择性. 如 Goto 小 组 ${ }^{[14]}$ 通过 1-特戊酰保护的吲哚丙酸 (21) 作为底物, 以较 高的产率和很好的 4-位选择性合成了 Uhle 酮衍生物 22
(图 4). 在这一反应中, 特戊酰的吸电子和大位阻效应降 低了 2-位的反应活性, 而另一方面, 外加的添加试剂氯 乙酰氯与 Lewis 酸三氯化铝作用形成的复合物可以络合 在特成酰胺上形成中间体，从而进一步阻碍了吲哚 2-位 的反应. Szántay 小组正是以 Uhle 酮衍生物 22 作为关键 前体，完成了麦角酸(2)和 cycloclavine (23) 的全合成 ${ }^{[15]}$.

\subsubsection{Pictet-Spengler 反应}

前面已经提到过，吲哚的 4-位不是活性最高的位 点, 而在吲哚苯环部分的 5-位或/和 7-位引入给电子取 代基可以增加苯环部分的电性，进而提高吲哚 4-位的反 应活性和选择性 ${ }^{[16]}$. 例如 5-差色胺类衍生物 24 可以与 相应的醛发生 Pictet-Spengler 反应, 得到在 2-位和 4-位 关环的产物，而其选择性与反应的 $\mathrm{pH}$ 值有关，酸性条 件下以 2-位关环产物为主，碱性条件下则以 4-位关环产 物为主.

Ishikura 小组 ${ }^{[17]}$ 正是利用这一反应完成了天然产物 aurantioclavine 的全合成. $N$-苠基保护的 5-羟色胺(27)可 以与醛在碱性条件下选择性的在 4-位关环，以 $60 \%$ 的收 率合成整个天然产物的分子骨架. 而后在钯/碳、甲酸铵 的作用下脱除 $\mathrm{N}$-苠基和 $\mathrm{O}$-三氟甲磺酰基，从而得到天 然产物 aurantioclavine (29), 而仅脱除 $N$-苠基的副产物 30 也可以在一定条件下转化为天然产物(图 5).

\subsection{3 分子内的 Michael 加成}

Bergman 小组 ${ }^{[18]}$ 在对 3-位 $\alpha, \beta$-不饱和酰基取代的吲 哚反应性质研究时发现, 吲哚衍生物 31 可以在三氯化 铝/氯化钠高温作用下，4-位发生分子内 Michael 加成反 应, 得到 3,4-桥环吲哚化合物 32 (图 6). 这一结果也证明 了在吲哚 3-位有强吸电子基取代时, 无需封闭其他活性 位点，就可以选择性地在 4-位发生亲电反应.
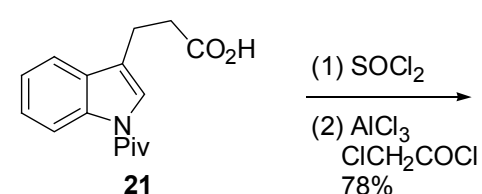

$78 \%$
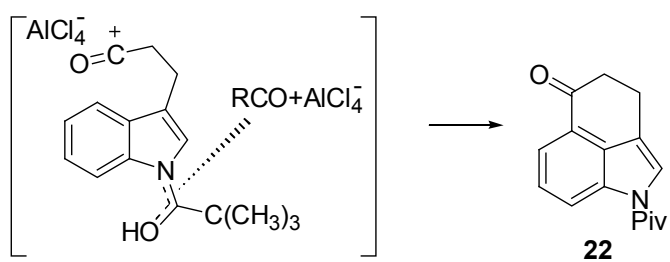

22

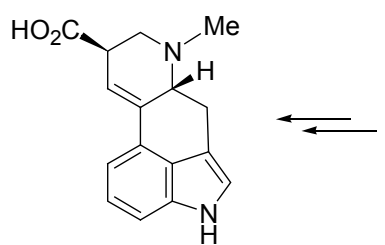

lysergic acid (2)

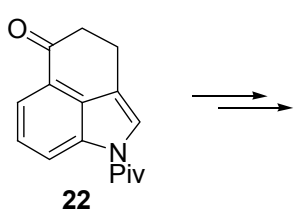

图 4 吲哚 4-位的 Friedel-Crafts 反应

Figure 4 Friedel-Crafts reaction on the 4-position of indoles 


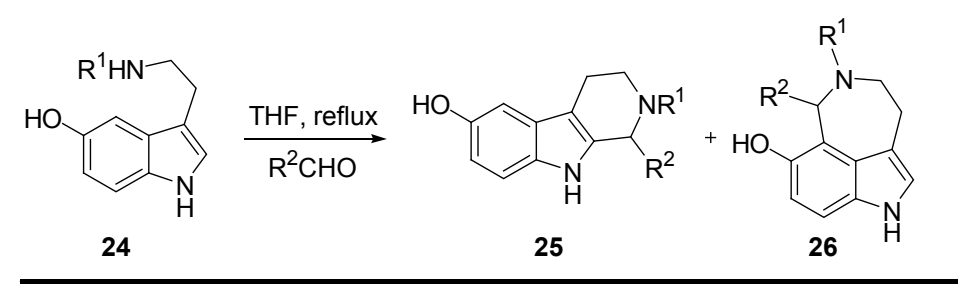<smiles>Oc1ccc2[nH]cc(CCNBr)c2c1</smiles>

27

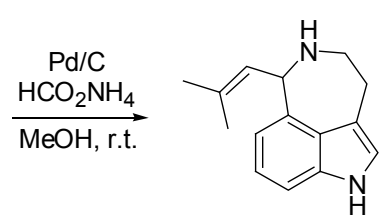

aurantioclavine (29)

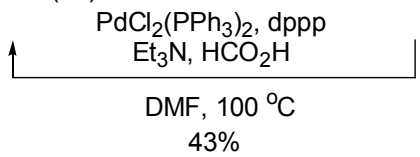

图 5 5-位取代吲哚 4-位的 Pictet-Spengler 反应

Figure 5 Pictet-Spengler reaction on the 4-position of 5-substituted indoles<smiles>CC(C)=CC(=O)c1c[nH]c2ccccc12</smiles>

31

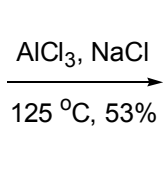

$25^{\circ} \mathrm{C}, 53 \%$<smiles>CC1(C)CC(=O)c2c[nH]c3cccc1c23</smiles>

32
图 6 吲哚 4-位的 Michael 加成反应

Figure 6 Michael addition on the 4-position of indoles

\subsubsection{Witkop 光环化反应}

合成 3,4-桥环吲哚还有一个重要的方法就是应用 Witkop 光环化反应(图 7). 1966年, Witkop 等 ${ }^{[19]}$ 首次报道 了在紫外照射下, $N$-氯乙酰- $L$-色氨酸(33) 可以在水溶液 中转化为吲哚 3,4-位桥连的八元内酰胺衍生物(36). 该 方法最大的特点是不需要使用任何保护基, 并且这一方 法还能够有效地用于合成吲哚苯环上有取代的桥环化 合物 ${ }^{[20]}$.

进一步通过对吲哚以及其他一些富电性芳环的光 环化反应进行荧光淬灭、溶剂效应、闪光光解以及动力 学等的研究表明, 此反应的机理很可能涉及到光激发的 分子内电子转移, 或者是激发的单线态富电性芳环与缺 电性的氯乙酰基团形成激态复合物, 然后形成一个双自 由基阳离子中间体, 最后通过自由基偶联在吲哚的 4-位 形成碳碳键, 从而实现了吲哚 4-位的直接官能团化.

Moody 小组 ${ }^{[21]}$ 通过进一步研究发现, 当增加底物羰 基 $\alpha$-位氯原子的数量时, 新增的氯原子可以通过稳定自
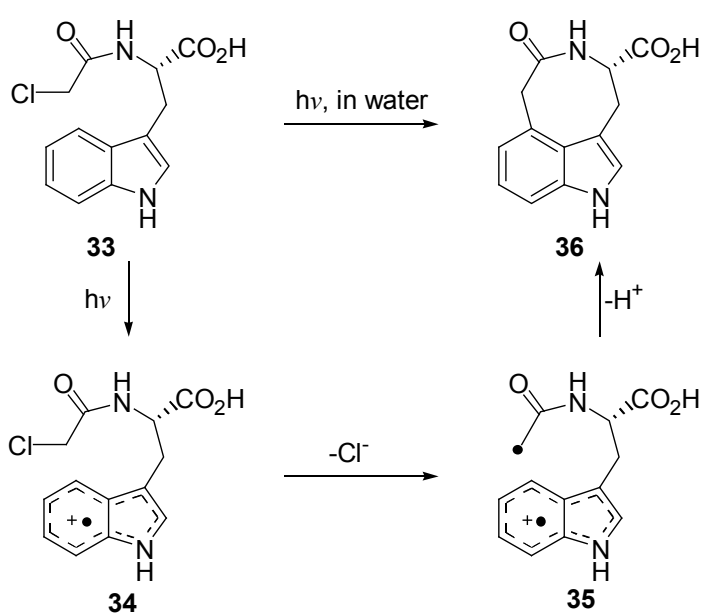

图 7 Witkop 光环化反应

Figure 7 Witkop photocyclization

由基中间体，从而提高光环化反应的产率，他们也利用 这一反应完成了天然产物 indolactam $\mathrm{V}$ 的全合成.

Feldman 小组 ${ }^{[22]}$ 在对天然产物 dragmacidin E 的全合 成中也利用了这一反应(图 8). 他们从羰基 $\alpha$-位二氯取 代的色氨酸甲酯衍生物(35)出发, 以 Witkop 光环化作为 关键反应得到了吲哚 3.4-并八元内酰胺环化合物 38, 并 经过多步转化, 成功构建了 dragmacidin E左边部分的骨 架结构(39), 进而最终实现了 dragmacidin $\mathrm{E}$ 的全合成. 


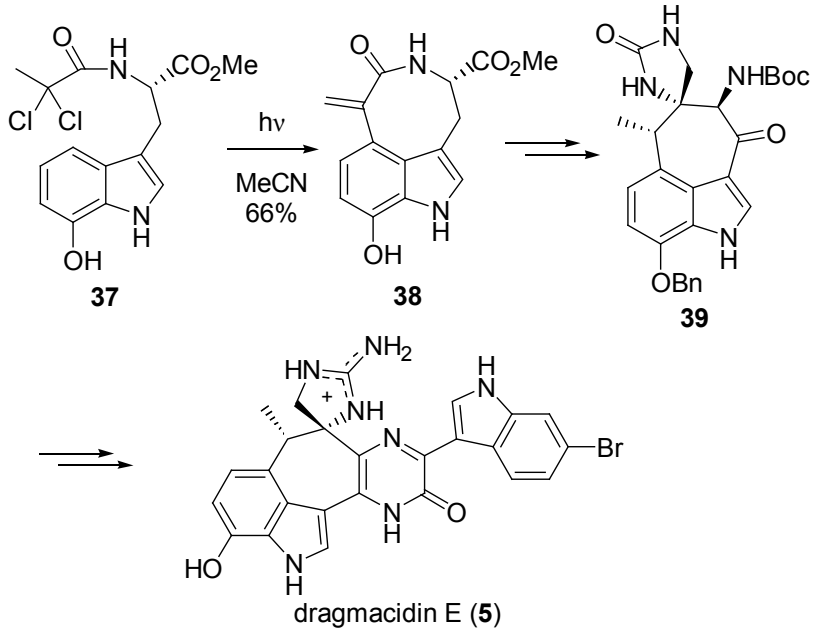

图 8 Feldman 小组通过 Witkop 光环化实现的 dragmacidin E 的全合成

Figure 8 Feldman's total synthesis of dragmacidin E based on Witkop photocyclization

Harran 小组 ${ }^{[23]}$ 也是利用这一反应, 在天然产物 diazonamide A 的全合成中成功构筑了右边的大环(图 9). 他们的合成从 7-溴色氨酸甲酯(40)开始, 经过几步转化 快速合成化合物 41, 再经与 7-差基色胺(42)缩合得到化 合物 43, 后者经乙酰基保护, 茮位氧化, 脱水环合后得 到化合物 44, 再利用 Witkop 光环化作为关键反应构建 出整个分子的骨架 45 . 而后经过还原, 氯代等几步简单 转化最终完成了 diazonamide A 的全合成. 同时他们在 研究中发现, 在吲哚的 7-位引入给电子的羟基可以提高 Witkop 光环化反应的产率, 从而大大提高了合成的效
率.

我们小组 ${ }^{[7 b]}$ 也将这一方法应用到了天然产物 decursivine 的全合成上(图 10). 我们从胡椒醇(46)出发, 经过溴代、偶联、水解、酰胺化等 4 步简单的转化得到 化合物 47, 最后利用一步串联的 Witkop 光环化/氯消除/ 氧加成反应作为关键反应，仅用 5 步就完成了天然产物 decursivine (6)消旋体的全合成. 整个合成路线无保护 基，且操作方便，简洁而高效. 采用同样的策略，我们 也完成了天然产物 serotobenine (50)消旋体的全合成， 并合成了一系列类似物, 而这些化合物很可能成为发现 抗疮药的重要先导化合物. 在此基础上, 我们 ${ }^{[7 \mathrm{c}]}$ 还完成 了 decursivine 的手性全合成.

\section{2 吲哚 3-位没有取代基在吲哚 3-位关环}

\subsection{1 分子内的 Friedel-Crafts 反应}

4-位取代的吲哚也可以在 3-位发生分子内 FriedelCrafts 反应，从而在 3-位关环，得到 3,4-桥环吲哚. Williams 小组 ${ }^{[24]}$ 在天然产物 Hapalindoles $\mathrm{J}$ 和 $\mathrm{U}$ 的全合成中, 就是利用了这一反应作为构建分子骨架的关键反应(图 11). 化合物 51 和 52 在氯化锡的促进下可发生反应得到 化合物 53, 后者在吲哚 3-位关环得到关键中间体 54, 从 而构筑出整个分子的骨架. 化合物 54 经过不同的几步 转化就可以分别得到天然产物 Hapalindoles $\mathrm{J}(\mathbf{5 5})$ 和 U (56).

\subsection{2 分子内的 Michael 加成}

Garg 小组 ${ }^{[9 c]}$ 在天然产物 indolactam $\mathrm{V}$ 的全合成中, 利用吲哚 3-位对 $\alpha, \beta$-不饱和酯的分子内 Michael 加成作

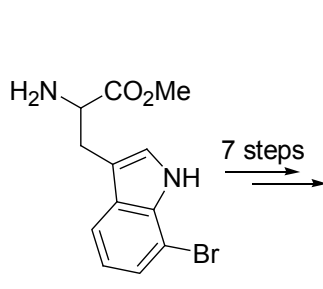

40
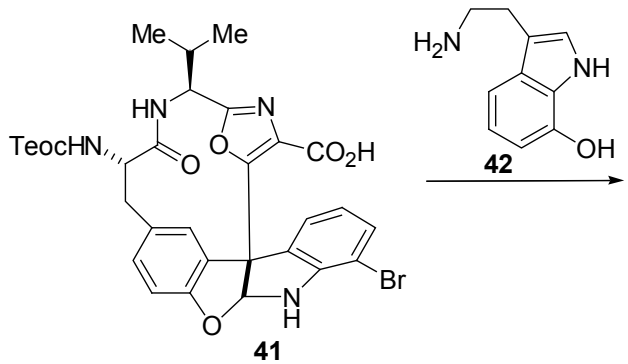

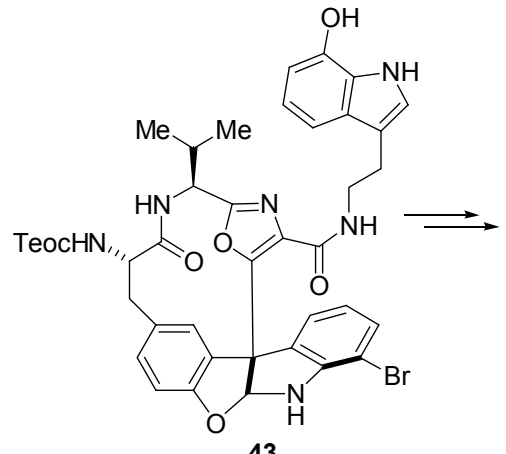

43
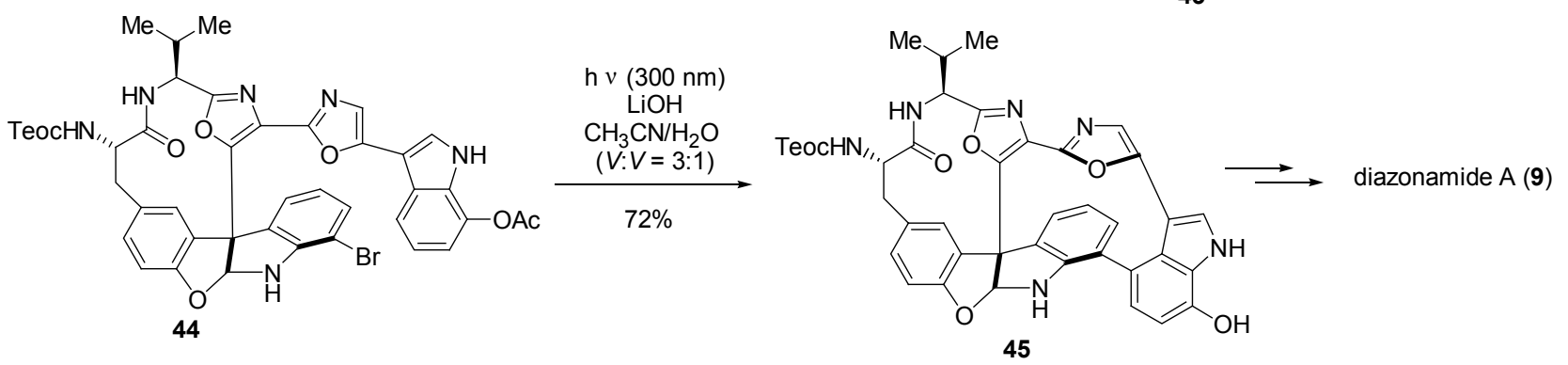

图 9 Harran 小组通过 Witkop 光环化实现的 diazonamide A 的全合成

Figure 9 Harran's total synthesis of diazonamide A based on Witkop photocyclization 


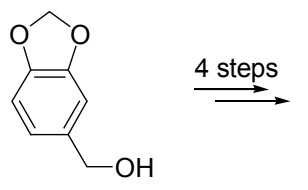

46

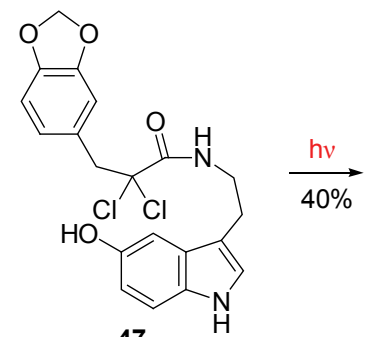

47

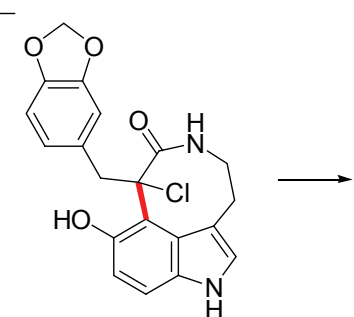

48

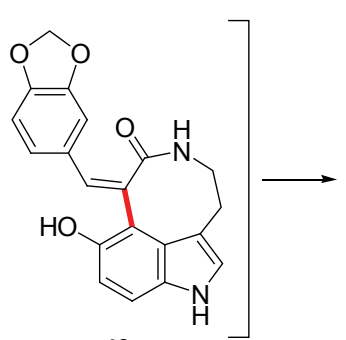

49

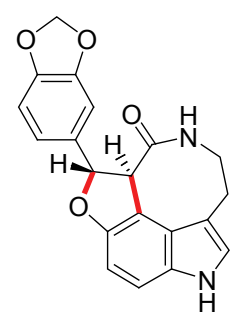

( \pm )-decursivine (6)

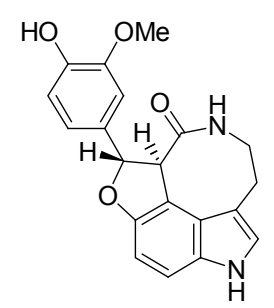

( \pm )-serotobenine $(\mathbf{5 0})$

图 10 贾小组通过 Witkop 光环化实现的 decursivine 的全合成

Figure 10 Jia's total synthesis of decursivine based on Witkop photocyclization<smiles>CC(C)(O)c1cccc2c1ccn2S(C)(=O)=O</smiles>

51<smiles>C=CC1C(OC)=CCCC1(C)C</smiles>

52<smiles>C=CC1(C)CCC(C(C)(C)c2cccc3c2ccn3S(C)(=O)=O)C(=O)C1OC</smiles>

53

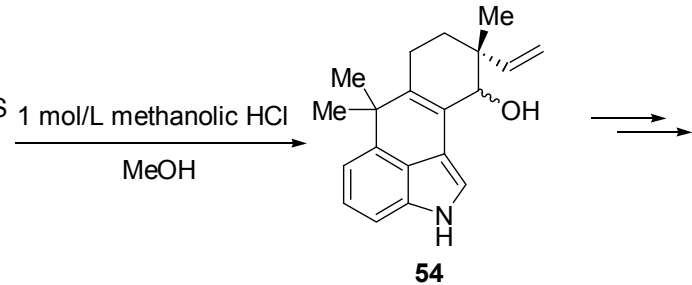

54

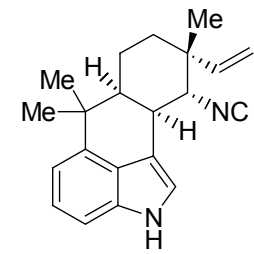

Hapalindole J (55)

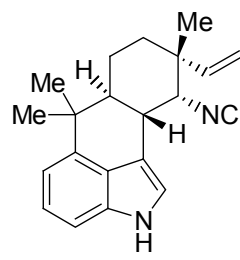

Hapalindole U (56)

图 11 Williams 小组通过分子内的 Friedel-Crafts 反应实现的 Hapalindoles $\mathrm{J}$ 和 $\mathrm{U}$ 的全合成

Figure 11 Williams' total synthesis of Hapalindoles $\mathrm{J}$ and $\mathrm{U}$ based on intramolecular Friedel-Crafts reaction

为关键反应完成了吲哚 3,4-桥环的构建. 他们通过化合 物 57, 利用吲哚炔的加成反应得到化合物 59, 再经脱 澳, 乙酰化, 消除得到关环前体 $\mathbf{6 0}$. 通过四氯化锆促进 的分子内的 Michael 加成反应构建出整个分子骨架 61, 同时很好地控制了酯基的手性. 最后经文献已知的转化 完成了天然产物 indolactam V (7)的全合成(图 12).

\section{3 吲哚 3-位和 4-位都有取代基利用适当的反应构建}

\section{3,4-桥环}

\subsection{1 分子内的 Heck 反应}

随着过渡金属催化反应的发展，并且由于其反应温
和及底物兼容性高等特点, 有机合成化学家们越来越多 地将过渡金属催化的反应用于合成中，也由此发展了一 些合成 3,4-桥环吲哚的策略 ${ }^{[25]}$, 分子内的 Heck 反应就 是其中的一个. 我们小组 ${ }^{[3 a]}$ 在麦角酸的全合成中就是利 用了分子内 Heck 反应作为关键反应完成了整个分子骨 架的构建. 从 4-碘色氨酸甲酯衍生物 62 出发, 经过几步 简单转化合成化合物 63. 利用烯烃复分解反应构建出上 面的六元环(64), 再经双键位移后得到化合物 65. 化合 物 65 能够顺利的发生分子内 Heck 反应，从而构建出整 个分子的骨架(66), 最后将甲酯水解同时 Boc 脱除, 完 成了麦角酸(2)的不对称全合成(图 13). 

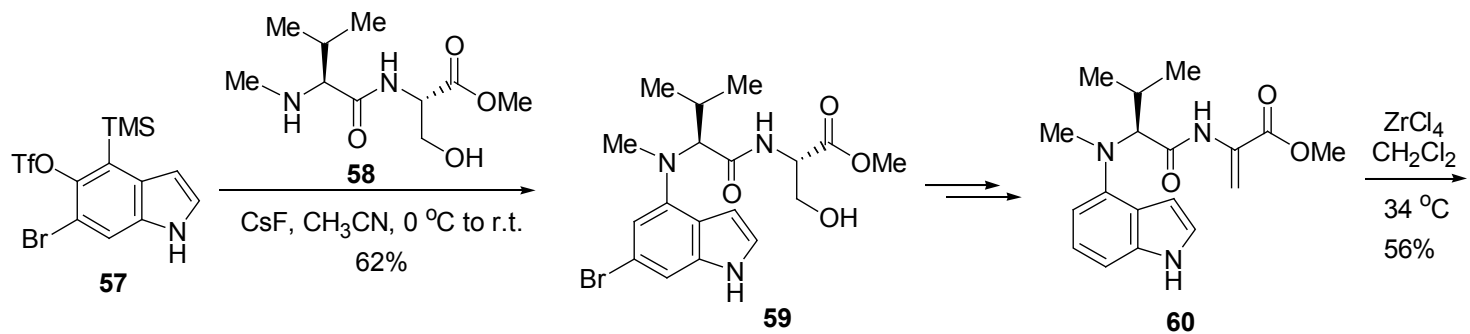<smiles>COC(=O)C(Cc1c[nH]c2cccc(Br)c12)NC(=O)C(C(C)C)N(C)C</smiles><smiles>CC(C)C(C(=O)N[C@@H](CO)Cc1c[nH]c2cccc([N+]([O-])([O-])[O-])c12)N(C)C</smiles>

图 12 Garg 小组通过分子内的 Michael 加成实现的 indolactam V 的全合成

Figure 12 Garg's total synthesis of indolactam V based on intramolecular Michael addition

Baran 小组 ${ }^{[26]}$ 以分子内的还原 Heck 反应作为关键 反应完成了天然产物 Hapalindole U 和 Ambiguine $\mathrm{H}$ 的全 合成(图 14). 他们利用取代的环已酮 67 和 4-澳吲哚 68 反应生成化合物 69 , 化合物 69 发生分子内还原 Heck 反 应, 构建出整个分子骨架 (70). 化合物 70 经过两步简单 转化即可得到天然产物 Hapalindole U (71), 而 Hapalindole $U$ 再经两步转化就得到了天然产物 Ambiguine H (72).

\subsection{2 烯烃复分解}

烯烃复分解反应是有机合成中构建大环最常用的 方法之一. 当在吲哚母核 3-位和 4-位分别引入适当的取 代基后, 利用烯烃复分解( $\mathrm{RCM}$ )反应在中间关环也可以 构建 3,4-桥环吲哚. Snieckus 等 ${ }^{[25 e]}$ 正是利用烯烃复分解 反应成功构筑了 $\mathrm{C} / \mathrm{D}$ 裂环麦角类生物碱的骨架结构, 为 合成吲哚 3,4-并大环的结构提供了一种有效的方法(图
15). 从 4-溴吲哚 68 出发, 经文献已知的方法合成化合 物 73, 再经 Stille 偶联、四氢铝锂还原、乙酰基保护以 及 $N$-烯丙基化得到关环前体 $\mathbf{7 5}$, 最后利用一步 RCM 得 到 3,4-桥环吲哚骨架(76).

\subsection{3 分子内的 $\mathrm{S}_{\mathrm{RN}} 1$ 反应}

Martin 小组 ${ }^{[27]}$ 在天然产物 Rugulovasines $\mathrm{A}$ 和 B 的 全合成中，以 $\mathrm{S}_{\mathrm{RN}} 1$ 反应作为关键的关环反应实现了目 标分子骨架的构筑(图 16). 他们从 4-溴吲哚(68)出发, 经三步简单转化合成化合物 77, 经 DIBAL-H 将氧基还 原成醛基，并通过一锅串联的插烯 Mannich 反应，在吲 哚 3-位引入呋喃片段, 得到化合物 78. 光照条件下, 在 叔丁醇钾和液氨存在下，化合物 $\mathbf{7 8}$ 可以发生关环得到 79. 在进一步研究中他们发现, 若无光照, 则不能得到理 想的产物，因此这一反应被认为是按照 $\mathrm{S}_{\mathrm{RN}} 1$ 反应的机
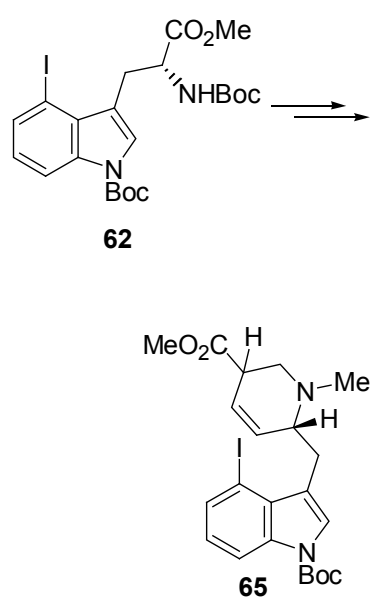

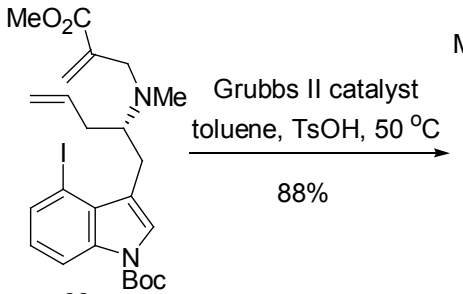

63
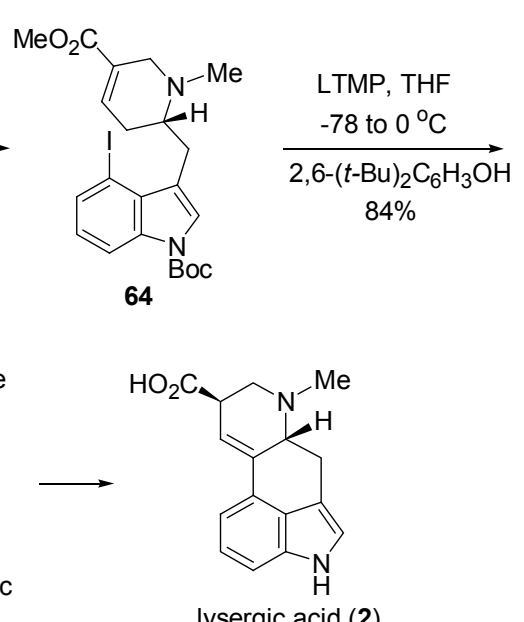

图 13 贾小组通过分子内 Heck 反应实现的麦角酸的全合成

Figure 13 Jia's total synthesis of lysergic acid based on intramolecular Heck reaction 
<smiles>C=CC1CC[C@@](C)(C=O)C(=O)C1</smiles>

67<smiles>Brc1cccc2[nH]ccc12</smiles>

68

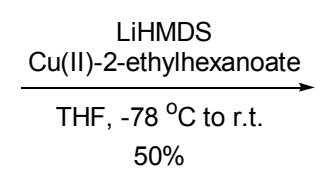

$50 \%$<smiles>C=CC1(C)CC2C(=O)C1CC2c1c[nH]c2cccc(C(C)(C)C)c12</smiles>

70<smiles>C=CC1CC(C)C(C(=O)c2c[nH]c3cccc(Br)c23)C(C)C1C</smiles>

69

\section{$\left[\mathrm{Pd}\left(\mathrm{P}(\mathrm{o}-\mathrm{tol})_{3}\right) \mathrm{OAc}\right]_{2}$ $\mathrm{NaOCHO}$ \\ TBAB, $\mathrm{Et}_{3} \mathrm{~N}, \mathrm{DMF}, 80^{\circ} \mathrm{C}$}

$65 \%$

2 steps

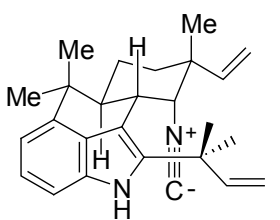

Ambiguine $\mathrm{H}$ (72)

图 14 Baran 小组通过分子内还原 Heck 反应实现的 Hapalindole U 和 Ambiguine H 的全合成

Figure 14 Baran's total synthesis of hapalindole $U$ 和 ambiguine $\mathrm{H}$ based on intramolecular reductive Heck reaction<smiles>C[R17](Br)c1cc2c(Br)cccc2[nH]1</smiles>

68<smiles>CCc1c[nH]c2cccc(Br)c12</smiles>

73
(1) AllylSnBu $3, \mathrm{Pd}\left(\mathrm{PPh}_{3}\right)_{4}$ Toluene, $110^{\circ} \mathrm{C}$

(2) $\mathrm{LiAlH}_{4}, \mathrm{Et}_{2} \mathrm{O} / \mathrm{THF}$, r.t.

(3) $\mathrm{Ac}_{2} \mathrm{O}$, pyridine, r.t. $41 \% 3$ steps<smiles></smiles><smiles>C=CCc1cccc2[nH]cc(CCN)c12</smiles>

74<smiles></smiles>

76
(1) $\mathrm{Boc}_{2} \mathrm{O}, \mathrm{NEt}_{3}, \mathrm{DMAP}$ (2) $n$-BuLi, AllylBr
THF, $-78{ }^{\circ} \mathrm{C}$ to r.t. $47 \% 3$ steps

图 15 Snieckus 小组通过烯烃复分解反应构建 3,4 桥环吲哚

Figure 15 Snieckus' construction of 3,4-fused indole based on RCM reaction

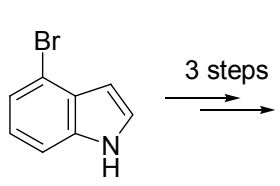

68<smiles>N#CCc1c[nH]c2cccc(Br)c12</smiles>

77<smiles>CC(C)(C)[Mg]Br</smiles>

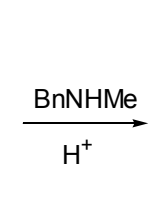

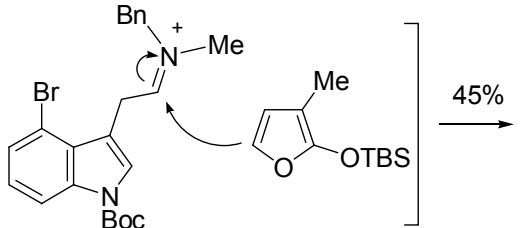

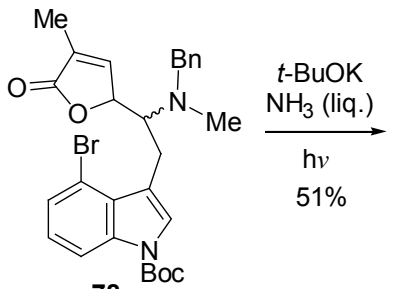

78<smiles>CCCCC1(C)Cc2c[nH]c3cccc(c23)[C@@]12C=CC(=O)O2</smiles>

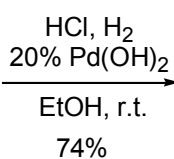

$74 \%$<smiles>CC1=C[C@]2(OC1=O)c1cccc3[nH]cc(c13)C[C@@]2(C)N</smiles>

Rugulovasines A \& B (80)

图 16 Martin 小组通过分子内 $\mathrm{S}_{\mathrm{RN}} 1$ 反应实现的 Rugulovasines A 和 $\mathrm{B}$ 的全合成

Figure 16 Martin's total synthesis of Rugulovasines A and B based on intramolecular $\mathrm{S}_{\mathrm{RN}} 1$ reaction

理进行的. 最后将茮基脱除即得到天然产物 Rugulovasines A 和 B (80).

\subsection{4 分子内插烯 Mannich 反应}

Martin 小组 ${ }^{[28]}$ 在之前对 Rugulovasines A 和 B 的全 合成中, 利用了分子间的插烯 Mannich 反应引入了吲哚
3-位的呋喃环片段. 在进一步的研究中, 他们尝试了分 子内的插烯 Mannich 反应, 并以此为关键反应实现了 Rugulovasines A 和 B 更加高效的全合成(图 17). 取代的 呋喃 81 与 4-溴吲哚衍生物 73 发生 Stille 偶联得到化合 物 82, 将氧基还原成醛基后紧接着进行一步分子内的插 


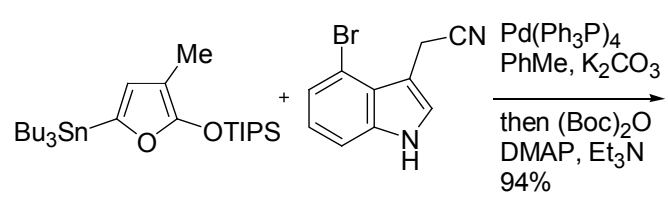

81

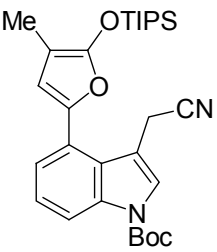

82

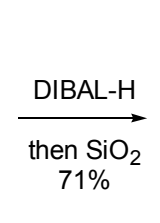

$1 \%$

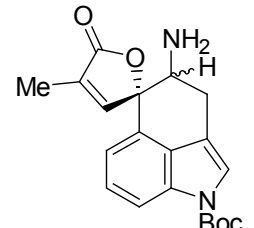

83

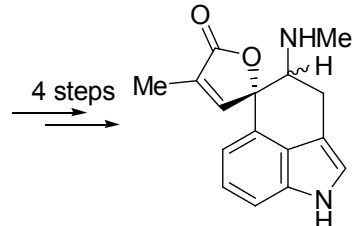

Rugulovasines A \& B (80)

图 17 Martin 小组通过分子内插烯 Mannich 反应实现的 Rugulovasines A 和 B 的全合成

Figure 17 Martin's total synthesis of rugulovasines A and B based on intramolecular vinylogous Mannich reaction

烯 Mannich 反应, 得到整个分子的骨架 $(\mathbf{8 3})$. 再经过几 步简单的转化, 就完成了天然产物 Rugulovasines A 和 B $(80)$ 的全合成.

\subsection{5 分子内 $\mathrm{S}_{\mathrm{N}} 2^{\prime}$ 反应}

我们小组 ${ }^{[29]}$ 在天然产物 clavicipitic acid 的全合成 中, 利用了高氯酸镁介导的 $\mathrm{S}_{\mathrm{N}} 2$ 反应实现了整个分子骨 架的构建(图 18). 从 4-氯色氨酸衍生物 84 出发, 经 Boc 保护, 芳基氯的 Heck 反应得到化合物 86, 通过高氯酸 镁促进的一锅串联的去保护/环合反应构筑出 3,4-桥环 吲哚的骨架 $(87)$, 再经脱除 Boc, 酯水解, 即完成了 clavicipitic acid (88) 的全合成.

\section{2 合成 3,4-桥环吲哚骨架的新策略}

如前文所述，传统的合成 3,4-桥环吲哚的方法都是 集中在 3,4-桥环的构筑上, 因此大部分都需要在吲哚的 4-位引入特定的取代基. 但是由于直接合成 4-位取代的 吲哚衍生物通常需要多步反应, 而直接官能团化吲哚的 4-位也存在很多困难，因此传统的策略都具有一定的局 限性. 发展通用的、可以简洁高效地构筑 3,4-桥环吲哚 的新方法对于有机合成化学家来讲一直是一个挑战. 近 几年来, 有机合成化学家们采用完全不同的切断方式与 合成策略, 将整个母核打开后再重建, 发展了一些新的 合成 3,4-桥环吲哚的方法.

\section{$2.16 \pi-$ 电环化}

通常合成吲哚的方法多是由取代的苯环与适当化 合物反应构筑吡咯环部分，也有少数是从吡咯出发而构 建苯环部分, 而 $6 \pi$-电环化则不同于以往常规的方法, 为吲哚的合成提供了一个新的策略. Funk 小组 ${ }^{[30 a]}$ 报道 了利用 $6 \pi$-电环化反应构建 3,4-桥环吲哚的方法(图 19). 二烯化合物 89 与 2-碘环已烯酮经过 Stille 偶联得到三烯 化合物 90, 后者在加热的条件下可顺利发生 $6 \pi$-电环化, 得到化合物 91. 经 DDQ 氧化、三氟乙酸脱除 Boc 保护 基、还原胺化后得到的化合物 93 在加热条件下发生关 环即可得到 3,4-桥环吲哚(94). 他们在研究中还发现, 这 一方法对于合成不同位取代的 3,4-桥环吲哚, 以及吲哚 3,4-并六元、七元、八元环的结构都是十分有效的，从而 为构建复杂的 3,4-桥环吲哚骨架提供了一个有效的方 法. 通过这一策略, Funk 小组 ${ }^{[30 b]}$ 还完成了天然产物 dragmacidin E 骨架结构(97)的构建, 为其全合成奠定了 基础.

\section{2 分子内呋喃的 Diels-Alder 反应}

2009 年, Wipf 小组 ${ }^{[31 \mathrm{a}]}$ 报道了以微波促进的分子内 呋喃的 Diels-Alder 反应构建 4-取代吲哚和 3,4-桥环吲哚 的方法. 与以往不同的是，这一方法采用了一种全新的 切断方式, 将整个吲哚母核的骨架完全打开(图 20).<smiles>CC(=O)ON(C)C(Cc1c[nH]c2cccc(Cl)c12)C(C)=O</smiles><smiles>COc1ccc(OC)cc1</smiles><smiles></smiles>
86

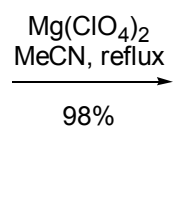<smiles>CC(=O)OCCCC(Cc1cn(Br)c2cccc(Cl)c12)C(C)=O</smiles><smiles>CC(C)(O)C=CC(=O)OCC(=O)OC(C)(C)C</smiles>
$75 \%$<smiles>CC(C)=CC1N[C@@H](C(=O)O)Cc2c[nH]c3cccc1c23</smiles>

clavicipitic acid (88)

图 18 贾小组通过分子内 $\mathrm{S}_{\mathrm{N}} 2^{\prime}$ 反应实现的 clavicipitic acid 的全合成

Figure 18 Jia's total synthesis of clavicipitic acid based on intramolecular $\mathrm{S}_{\mathrm{N}} 2$ ' reaction 


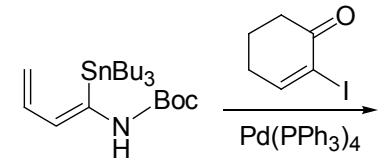

89 Cul, THF, $50{ }^{\circ} \mathrm{C}$ $81 \%$

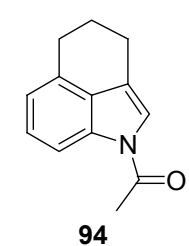

$$
\begin{aligned}
& \frac{\mathrm{Ac}_{2} \mathrm{O}, \mathrm{NEt}_{3}}{130{ }^{\circ} \mathrm{C}} \\
& 77 \% \\
& \text { (2 steps) }
\end{aligned}
$$<smiles>C/C=C\C=C(\N)C1=CCCCC1=O</smiles>

$110^{\circ} \mathrm{C}$

$90^{\mathrm{Boc}}$<smiles>Nc1cccc2c1C(=O)CCC2</smiles>

$91 \mathrm{Boc}$ $91 \% \mid \begin{aligned} & \mathrm{DDQ} \\ & 50^{\circ} \mathrm{C}\end{aligned}$<smiles>O=C(O)CNc1cccc2c1C(=O)CCC2</smiles>

(1) TFA, $94 \%$

(2) $\mathrm{NaCNBH}_{3}$ $\mathrm{OHCCO}_{2} \mathrm{H}$<smiles>O=C(Nc1cccc2c1C(=O)CCC2)OCc1ccccc1</smiles><smiles>[B]C1=C(C=C)N([Pb])C(C)(C)O1</smiles>

95

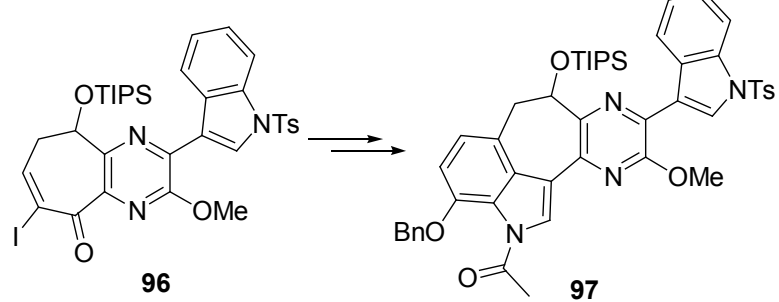

图 19 Funk 小组通过 $6 \pi$-电环化合成 dragmacidin E 骨架结构

Figure 19 Funk's synthesis of core structure of dragmacidin E based on $6 \pi$-electrocyclic ring closures
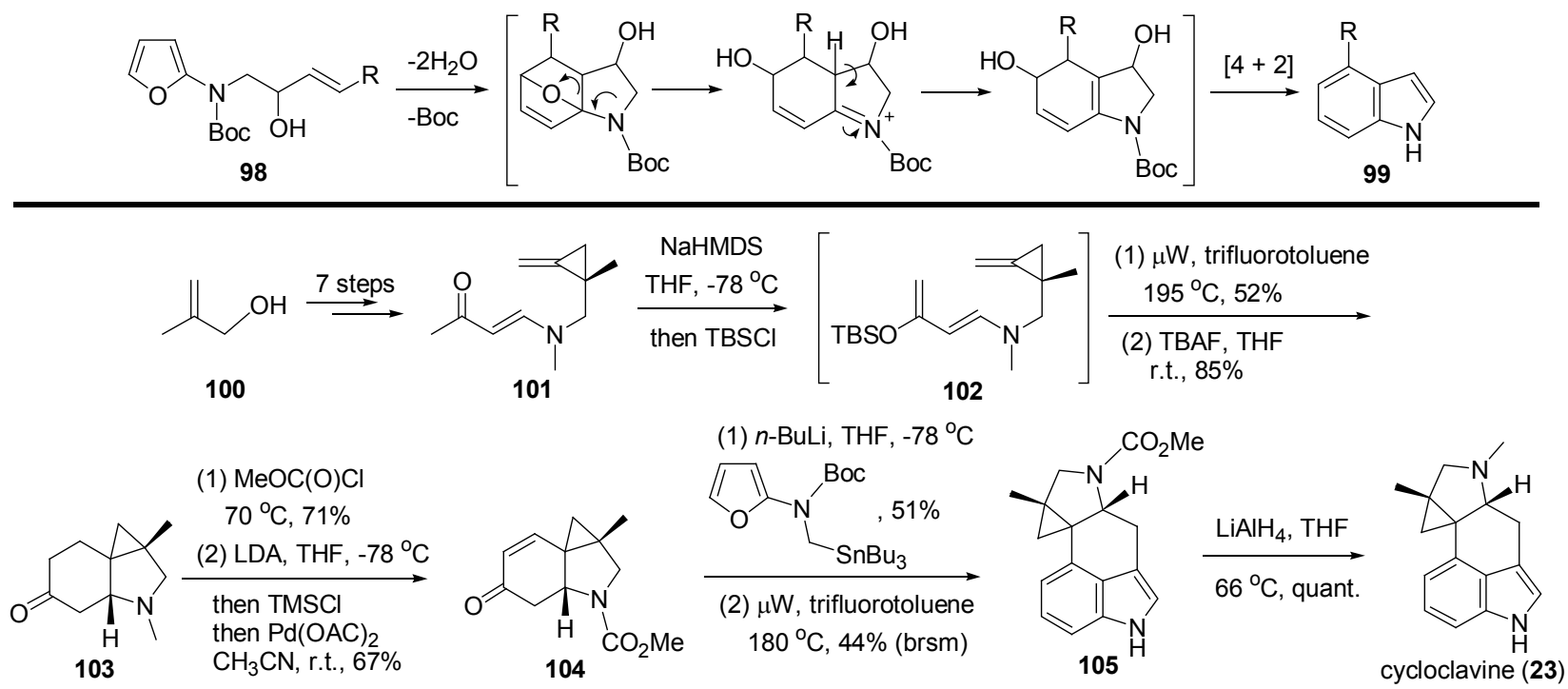

图 20 Wipf 小组通过分子内呋喃的 Diels-Alder 反应实现的 cycloclavine 的全合成

Figure 20 Wipf's total synthesis of cycloclavine based on intramolecular Diels-Alder cycloaddition of furan

2011 年, Wipf 小组 ${ }^{[31 b]}$ 以这一反应作为最后的关键 反应实现了天然产物 cycloclavine 的高效全合成. 他们 从 $\beta$-烯丙醇 $(100)$ 出发, 经几步简单转化得到化合物 101, 烯醇化后 TBS 保护，紧接着发生一步 Diels-Alder 反应再 用四丁基氟化铵将 TBS 脱除从而得到化合物 103. 将 $N$ 甲基转化为甲酯基后利用 Saegusa 氧化得到化合物 104. 与含呋喃的片段偶联后，在微波条件下发生分子内 Diels-Alder 反应以 $44 \%$ 的收率构筑出整个分子骨架
(105). 最后经四氢铝锂还原，完成了天然产物 cycloclavine (23)的全合成. 在引入所有基团后，将关键 反应放在最后，大大提高了整个合成的效率.

\section{3 分子内 Fischer 吲哚合成}

2012 年, Cho 小组 ${ }^{[32]}$ 报道了应用分子内的 Fischer 吲哚合成串 Claisen 重排的方法合成 3,4-桥环吲哚骨架 (图 21). 他们在对分子内 Fischer 吲哚合成反应的研究中 发现，虽然间位有潜在羰基取代的芳基肼不能发生分子 
内的 Fischer 反应，但对位取代的芳基肼却能顺利地进 行该反应，而当在侧链上加入一双键后，即可进一步发 生 Claisen 重排从而得到 3,4-桥环吲哚的骨架. 这一反应 的底物比较容易制备, 并且反应条件温和, 因此对于官 能团应该有很好的兼容性和耐受性，但该方法在天然产 物全合成中的应用还尚未见报道.

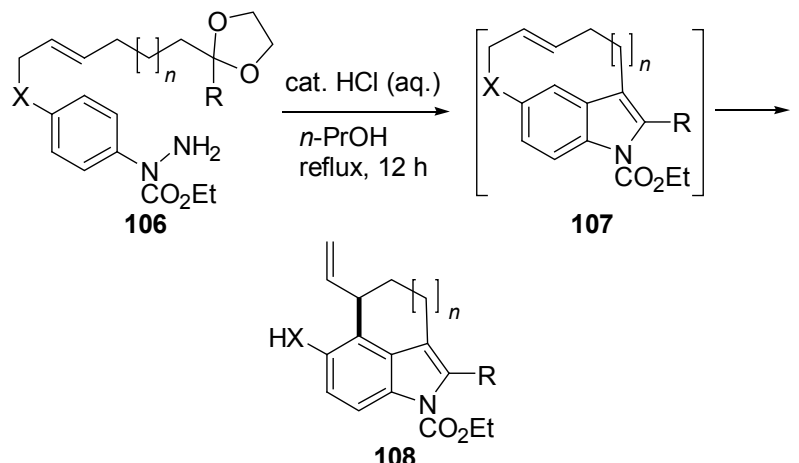

图 21 Cho 小组通过分子内 Fischer 吲哚合成构建 3,4-桥环吲 哚

Figure 21 Cho's construction of 3,4-fused indole based on intramolecular Fischer indole synthesis

\section{4 分子内 Larock 吲哚合成}

我们小组 ${ }^{[3 a, 7 b, 7 c, 9 a, 29,33]}$ 在合成了一系列含有 3,4-桥环 吲哚骨架的天然产物之后，也一直在探索一种通用而高 效的构建这类骨架的方法. 在研究中我们发现, 卤原子 邻位取代的邻卤苯胺能够以分子内的形式发生 Larock 吲哚合成反应，一步构筑两个环，从而得到 3,4-桥环吲 哚骨架. 进一步的研究表明，这一反应对于环上不同原 子取代, 以及不同环系大小, 其兼容性都是很好的, 并
且在合成吲哚 3,4-并中环或大环时，也能给出中等甚至 较高的收率. 我们 ${ }^{[34]}$ 在 2013 年初报道了这一合成 3,4桥环吲哚的新策略，并以此作为关键反应，实现了天然 产物 fargesine 的首次全合成. 我们从已知的醛 111 和胺 112 出发, 通过还原胺化, Boc 保护得到化合物 114, 将 硝基还原后即得到取代的邻碘苯胺(115)这一关环前体， 经分子内的 Larock 反应可以以基本定量的产率成功构 建出整个分子的骨架结构(116). 最后经脱保护，还原胺 化, $N$-氧化等 4 步简单转化，完成了 fargesine (117)的全 合成(图 22).

Boger 小组 ${ }^{[35]}$ 也于 2013 年初报道了与我们类似的 想法. 他们利用零价钯介导的分子内 Larock吅哚合成反 应，通过取代的邻溴苯胺，同样实现了 3,4-桥环吲哚的 构建，并且对更大的环系进行了考察(图 23).

\section{3 总结与展望}

本文概述了构建 3,4-桥环吲哚骨架的传统常用方法 和近年来发展的一些新策略，以及这些方法在天然产物 全合成中的应用. 天然产物复杂多样的结构促使我们为 其合成发展简洁高效的方法和策略，而这些方法和策略 只有能真正应用于合成中才能更加体现其价值. 随着越 来越多的吲哚类生物碱被分离鉴定，快速而有效的方法 和策略是合成这些天然产物及其类似物并对它们进行 活性篎选的前提和保障. 相信在以后的研究中会有更多 合成 3,4-桥环吲哚骨架的策略发展起来，以克服目前一 些方法的局限性, 从而为药物化学研究提供重要的先导 化合物.
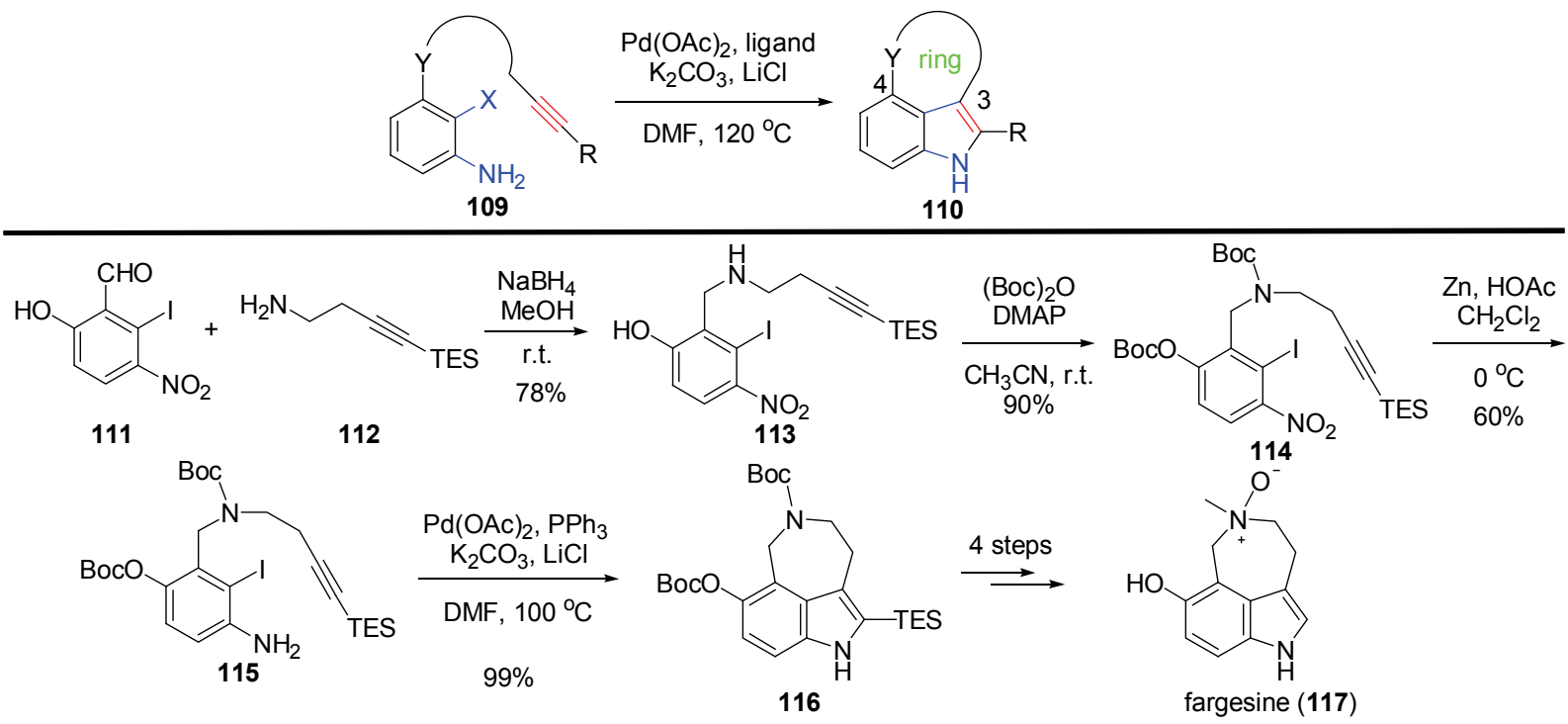

图 22 贾小组通过分子内 larock 吲哚合成实现的 fargesine 的全合成

Figure 22 Jia's total synthesis of fargesine based on intramolecular larock indole synthesis 


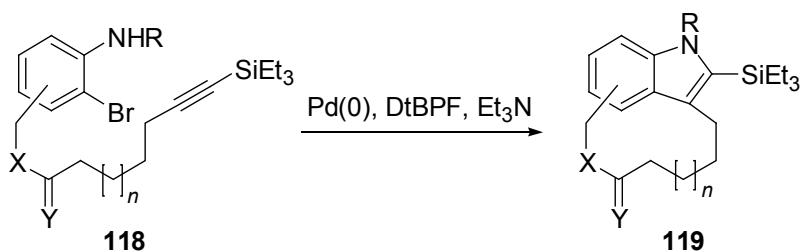

图 23 Boger 小组通过分子内 Larock 吲哚合成构建 3,4-桥环 吲哚

Figure 23 Boger's construction of 3,4-fused indole based on intramolecular Larock indole synthesis

\section{References}

[1] For recent reviews on the synthesis of indoles, see: (a) Humphrey, G. R.; Kuethe, J. T. Chem. Rev. 2006, 106, 2875.

(b) Cacchi, S.; Fabrizi, G. Chem. Rev. 2005, 105, 2873.

(c) Gribble, G. W. J. Chem. Soc., Perkin Trans. 1 2000, 1045.

[2] For total synthesis of dehydrobufotenine, see: (a) Peat, A. J.; Buchwald, S. L. J. Am. Chem. Soc. 1996, 118, 1028.

(b) Jackson, B.; Hester, J. J. Org. Chem. 1964, 29, 1158.

[3] For total synthesis of lysergic acid, see: (a) Liu, Q.; Jia, Y. Org. Lett. 2011, 13, 4810.

(b) Iwata, A.; Inuki, S.; Oishi, S.; Fujii, N.; Ohno, H. J. Org. Chem. 2011, 76, 5506.

(c) Kurokawa, T.; Isomura, M.; Tokuyama, H.; Fukuyama, T. Synlett 2009, 775.

[4] For total synthesis of $N$-methylwelwitindolinone, see: (a) Huters, A. D.; Quasdorf, K. W.; Styduhar, E. D.; Garg, N. K. J. Am. Chem. Soc. 2011, 133, 15797.

(b) Bhat, V.; Allan, K. M.; Rawal, V. H. J. Am. Chem. Soc. 2011, 133, 5798 .

[5] For total synthesis of communesin F, see: (a) Yang, J. Wu, H.; Shen, L.; Qin, Y. J. Am. Chem. Soc. 2007, 129, 13794.

(b) Zuo, Z.; Xie, W.; Ma, D. J. Am. Chem. Soc. 2010, 132, 13226.

(c) Liu, P.; Seo, J. H.; Weinreb, S. M. Angew. Chem., Int. Ed. 2010, 49, 2000

(d) Belmar, J.; Funk, R. L. J. Am. Chem. Soc. 2012, 134, 16941.

[6] For total synthesis of dragmacidin E, see: Feldman, K. S.; Ngernmeesri, P. Org. Lett. 2011, 13, 5704.

[7] For total synthesis of decursivine, see: (a) Mascal, M.; Modes, K. V.; Durmus, A. Angew. Chem., Int. Ed. 2011, 50, 4445.

(b) Qin, H.; Xu, Z.; Cui, Y.; Jia, Y. Angew. Chem., Int. Ed. 2011, 50, 4447.

(c) Hu, W.; Qin, H.; Cui, Y.; Jia, Y. Chem. Eur. J. 2013, 19, 3139.

(d) Sun, D.; Zhao, Q.; Li, C. Org. Lett. 2011, 13, 5302.

(e) Leduc, A. B.; Kerr, M. A. Eur. J. Org. Chem. 2007, 237;

(f) Koizumi, Y.; Kobayashi, H.; Wakimoto, T.; Furuta, T.; Fukuyama, T.; Kan, T. J. Am. Chem. Soc. 2008, 130, 16854.

[8] For total synthesis of penitrem D, see: Smith, III. A. B.; Kanoh, N.; Ishiyama, H.; Noriaki, M.; Rainier, J. D.; Hartz, R. A.; Cho, Y. S.; Cui, H.; Moser, W. H. J. Am. Chem. Soc. 2003, 125, 8228.

[9] For total synthesis of indolactam V, see: (a) Xu, Z.; Zhang, F.; Zhang, L.; Jia, Y. Org. Biomol. Chem. 2011, 9, 2512.

(b) Bronner, S. M.; Goetz, A. E.; Garg, N. K. Synlett 2011, 2599.

(c) Bronner, S. M.; Goetz, A. E.; Garg, N. K. J. Am. Chem. Soc. 2011, 133, 3832.

(d) Meseguer, B.; Alonso-D'iaz, D.; Griebenow, N.; Herget, T.; Waldmann, H. Angew. Chem., Int. Ed. 1999, 38, 2902.

(e) Moreno, O. A.; Kishi, Y. J. Am. Chem. Soc. 1996, 118, 8180.

[10] For total synthesis of diazonamide A, see: (a) Burgett, A. W. G.; Li,
Q.; Wei, Q.; Harran, P. G. Angew. Chem., Int. Ed. 2003, 42, 4961.

(b) Nicolaou, K. C.; Chen, D. Y.-K.; Huang, X.; Ling, T.; Bella, M.; Snyder, S. A. J. Am. Chem. Soc. 2004, 126, 12888.

(c) Nicolaou, K. C.; Hao, J.; Reddy, M. V.; Rao, P. B.; Rassias, G.; Snyder, S. A.; Huang, X.; Chen, D. Y.-K.; Brenzovich, W. E.; Giuseppone, N.; Giannakakou, P.; O'Brate, A. J. Am. Chem. Soc. 2004, 126, 12897.

(d) Knowles, R. R.; Carpenter, J.; Blakey, S. B.; Kayano, A.; Mangion, I. K.; Sinz, C. J.; MacMillan, D. W. C. Chem. Sci. 2011, 2, 308 .

(e) Cheung, C.-M.; Goldberg, F. W.; Magnus, P.; Russell, C. J.; Turnbull, R.; Lynch, V. J. Am. Chem. Soc. 2007, 129, 12320.

(f) Mai, C.-K.; Sammons, M. F.; Sammakia, T. Angew. Chem., Int. Ed. 2010, 49, 2397.

[11] (a) Kornfeld, E. C.; Fornefeld, E. J.; Kline, G. B.; Mann, M. J.; Morrison, D. E.; Jones, R. G.; Woodward, R. B. J. Am. Chem. Soc. 1956, 78, 3087.

(b) Kornfeld, E. C.; Fornefeld, E. J.; Kline, G. B.; Mann, M. J.; Jones, R. G.; Woodward, R. B. J. Am. Chem. Soc. 1954, 76, 5256.

[12] (a) Szmuszkovicz, J. J. Org. Chem. 1964, 29, 843.

(b) Nagasaka, T.; Ohki, S. Chem. Pharm. Bull. 1977, 25, 3023.

[13] (a) Nakatsuka, S. -i.; Miyazaki, H.; Goto, T. Tetrahedron Lett. 1980, $21,2817$.

(b) Lee, Y. S.; Min, B. J.; Park, Y. K.; Lee, J. Y.; Lee, S. J.; Park, H. Tetrahedron Lett. 1999, 40, 5569.

(c) Lee, Y. S.; Min, B. J.; Park, Y. K.; Lee, J. Y.; Lee, S. J.; Park, H. Tetrahedron 1999, 55, 12991.

[14] Teranishi, K.; Hayashi, S.; Nakatsuka, S.-I.; Goto, T. Tetrahedron Lett. 1994, 35, 8173.

[15] (a) Incze, M.; Dörnyei, G.; Moldvai, I.; Temesvári-Major, E.; Egyed, O.; Szántay, C. Tetrahedron 2008, 64, 2924.

(b) Moldvai, I.; Temesvári-Major, E.; Incze, M.; Szentirmay, É.; Gács-Baitz, E.; Szántay, C. J. Org. Chem. 2004, 69, 5993.

[16] Condie, G. C.; Channon, M. F.; Ivory, A. J.; Kumar, N.; Black, D. S. Tetrahedron 2005, 61, 4989.

[17] (a) Somei, M.; Seto, M. Heterocycles 2003, 60, 519.

(b) Somei, M.; Teranishi, S.; Yamada, K.; Yamada, F. Chem. Pharm. Bull. 2001, 49, 1159.

(c) Yamada, K.; Namerikawa, Y.; Haruyama, T.; Miwa, Y.; Yanada, R.; Ishikura, M. Eur. J. Org. Chem. 2009, 33, 5752.

[18] Bergman, J.; Venemalm, L.; Gogoll, A. Tetrahedron 1990, 46, 6067.

[19] (a) Yonemitsu, O.; Cerutti, P.; Witcop, B. J. Am. Chem. Soc. 1966, $88,3941$.

(b) Naruto, S.; Yonemitsu, O. Tetrahedron Lett. 1975, 16, 3399.

(c) Naruto, S.; Yonemitsu, O. Chem. Pharm. Bull. 1980, 28, 900.

[20] (a) Kobayashi, T.; Spande, T. F.; Aoyagi, H.; Witcop, B. J. Med. Chem. 1969, 12, 636 .

(b) Klohr, S. E.; Cassady, J. M. Synth. Commun. 1988, 18, 671.

[21] (a) Mascal, M.; Moody, C. J. J. Chem. Soc., Chem. Commun. 1988, 587.

(b) Mascal, M.; Moody, C. J. J. Chem. Soc., Chem. Commun. 1988, 589.

(c) Beck, A. L.; Mascal, M.; Moody, C. J.; Slawin, A. M. Z.; Williams, D. J.; Coates, W. J. J. Chem. Soc., Perkin Trans. 1 1992, 797. (d) Beck, A. L.; Mascal, M.; Moody, C. J.; Coates, W. J. J. Chem. Soc., Perkin Trans. 1 1992, 813.

(e) Mascal, M.; Moody, C. J.; Slawin, A. M. Z.; Williams, D. J. J. Chem. Soc., Perkin Trans. 1 1992, 823.

(f) Mascal, M.; Moody, C. J.; Morrell, A. I.; Slawin, A. M. Z.; Williams, D. J. J. Am. Chem. Soc. 1993, 115, 813.

(g) Mascal, M.; Wood, I. G.; Begley, M. J.; Batsanov, A. S.; Walsgrove, T.; Slawin, A. M. Z.; Williams, D. J.; Drake, A. F.; Sili- 
gardi, G. J. Chem. Soc., Perkin Trans. 1 1996, 2427.

[22] (a) Feldman, K. S.; Ngernmeesri, P. Org. Lett. 2005, 7, 5449.

(b) Feldman, K. S.; Ngernmeesri, P. Org. Lett. 2011, 13, 5704.

[23] (a) Li, J.; Jeong, S.; Esser, L.; Harran, P. G. Angew. Chem., Int. Ed. 2001, 40, 4765 .

(b) Burgett, A. W. G.; Li, Q.; Harran, P. G. Angew. Chem., Int. Ed. 2003, 42, 4961.

[24] Rafferty, R. J.; Williams, R. M. J. Org. Chem. 2012, 77, 519.

[25] (a) Baran, P. S.; Thomas, J.; Maimone, T. J.; Richter, J. M. Nature 2007, 446, 404.

(b) Xu, Q.; Dai, L.; You, S. Chem. Sci. 2013, 4, 97.

(c) Hellal, M.; Singh, S.; Cuny, G. D. J. Org. Chem. 2012, 77, 4123.

(d) Lim, H. J.; Gallucci, J. C.; RajanBabu, T. V. Org. Lett. 2010, 12, 2162.

(e) Kalinin, A. V.; Chauder, B. A.; Rakhit, S.; Snieckus, V. Org. Lett. 2003, 5, 3519.

(f) Katayama, S.; Ae, N.; Nagata, R. J. Org. Chem. 2001, 66, 3474.

(g) Horwell, D. C.; Nichols, P. D.; Ratcliffe, G. S.; Roberts, E. J. Org. Chem. 1994, 59, 4418.

(h) Peshkov, V. A.; Hove, S. V.; Donets, P. A.; Pereshivko, O. P.; Hecke, K. V.; Meervelt, L. V.; Van der Eycken, E. V. Eur. J. Org. Chem. 2011, 1837.

(i) Söderberg, B. C. G.; Hubbard, J. W.; Rector, S. R.; O'Neil, S. N. Tetrahedron 2005, 61, 3637.

(j) Söderberg, B. C. G.; Rector, S. R.; O'Neil, S. N. Tetrahedron Lett. 1999, 40, 3657.
[26] Baran, P. S.; Maimone, T. J.; Richter, J. M. Nature 2007, 446, 404.

[27] Martin, S. F.; Liras, S. J. Am. Chem. Soc. 1993, 115, 10450.

[28] Liras, S.; Lynch, C. L.; Fryer, A. M.; Vu, B. T.; Martin, S. F. J. Am. Chem. Soc. 2001, 123, 5918.

[29] Xu, Z.; Li, Q.; Zhang, L.; Jia, Y. J. Org. Chem. 2009, 74, 6859.

[30] (a) Greshock, T. J.; Funk, R. L. J. Am. Chem. Soc. 2006, 128, 4946. (b) Huntley, R. J.; Funk, R. L. Org. Lett. 2006, 8, 4775.

[31] (a) Petronijevic, F.; Timmons, C.; Cuzzupe, A.; Wipf, P. Chem. Commun. 2009, 104.

(b) Petronijevic, F.; Wipf, P. J. Am. Chem. Soc. 2011, 133, 7704.

[32] Park, I.-K.; Park, J.; Cho, C.-G. Angew. Chem., Int. Ed. 2012, 55, 2496.

[33] (a) Xu, Z.; Hu, W.; Zhang, F.; Li, Q.; Lu, Z.; Zhang, L.; Jia, Y. Synthesis 2008, 3981.

(b) Hu, C.; Qin, H.; Cui, Y.; Jia, Y. Tetrahedron 2009, 65, 9075.

(c) Xu, Z.; Li, Q.; Zhang, L.; Jia, Y. J. Org. Chem. 2009, 74, 6859.

(d) Hu, W.; Zhang, F.; Xu, Z.; Liu, Q.; Cui, Y.; Jia, Y. Org. Lett. 2010, 12, 956.

(e) Xu, Z.; Hu, W.; Liu, Q.; Zhang, L.; Jia, Y. J. Org. Chem. 2010, 75,7626 .

(f) Liu, Y.; Zhang, L.; Jia, Y. Tetrahedron Lett. 2012, 53, 684.

(g) Jiang, D.; Xu, Z.; Jia, Y. Tetrahedron 2012, 68, 4225.

[34] Shan, D.; Gao, Y.; Jia, Y. Angew. Chem., Int. Ed. 2013, 52, 4902.

[35] Breazzano, S. P.; Poudel, Y. B.; Boger, D. L. J. Am. Chem. Soc. 2013, 135, 1600 . 